\title{
A Navel approach Image compression based on Luminance and Chrominance using Binary Wavelet Transform (BWT) and Raster Line Technique
}

\author{
${ }^{1}$ G. Thippanna, ${ }^{2}$ Dr. T. Bhaskar Reddy \\ ${ }^{1,2}$ Research Scholar Dept of Computer Science and Technology, S.K. University, Anantapur.
}

\begin{abstract}
In rapid development multimedia the Images are awesome use full, image coding is the key and most prevalent in mass communication and storage system. This paper represents image compression based on Luminance and Chrominance using Binary Wavelet Transform ${ }^{l}$ and Raster Line technique is proposed. This method shows the results better on standard JPEG and RAW images and reconstructs the images.
\end{abstract}

Key Words: - Image compression, BWT, JPEG compression, Lossy and Lossless, Raster Line Techniques.

\section{Introduction:-}

In rapid development multimedia the Images are awesome use full, image coding is the key and most prevalent in mass communication and storage system. Here using Raster Line Technique ${ }^{6-9}$ along with Luminance and Chrominance of Lossy and Lossless ${ }^{2-5}$ techniques. In a distributed environment large image files remain a major bottleneck within systems. Compression is an important component of the solutions available for creating file sizes of manageable and transmittable dimensions. Increasing the bandwidth is another method, but the cost sometimes makes this a less attractive solution. Platform portability and performance are important in the selection of the compression/decompression technique to be employed. Compression solutions today are more portable due to the change from proprietary high end solutions to accepted and implemented international standards. In most of the applications the exact restoration of stored image is not mandatory. This fact can help to make the storage more effective, and in this way we get the lossy compression methods. JPEG is evolving as the industry standard technique for the compression of continuous tone images. But this has a limitation for the color images that the application where the color integrity is important like correcting chromatic aberration is not suitable for JPEG data. In this paper a lossy image compression is proposed by considering the chrominance aberration correction.

\section{Binary Wavelet Transform:-}

The wavelet-transform compression technique offers a better compression performance than the DCTbased JPEG compression standard. In addition, many features such as quality and resolution scalability can be achieved with a single bit stream. This would not be possible for the current JPEG. However, DCT-based JPEG has the clear advantage of a very low implementation complexity ${ }^{4}$ over the wavelet-based technique. Wavelets representation is suggested in many of the image applications like edge detection, image coding, filtering and time frequency analysis due to its fastness and convenient tree structures. Most of the existing wavelet filters designed in the real field for gray level images have wide range of wavelet coefficients and bring out an expansion in the alphabet size of the symbols, leads to extra passes and bits for representing sign information of the wavelet coefficients. This expansion dramatically increases the model cost of the entropy coder for gray level images which are represented as eight alphabets. The most important feature of the BWT is the conservation of alphabet size of wavelet coefficients, which indicates that the transformed images have the same number of greyscale levels as the original images. In particular, for a K-bit greyscale image, the range of BWT coefficients is still maintained within [0,2K-1]. Therefore, it is reasonable to expect that the compression efficiency of the BWT coefficients can be improved in that extra bits originally used to code sign information of the transform coefficients which are saved to code more significant coefficients. The compression complexity might be reduced as the BWT contains simple exclusive-or (XOR) operations only and a maximum number of eight coding passes are involved during the encoding procedure ${ }^{1}$. 
Proposed Method:-

The proposed method is shown in figure 1

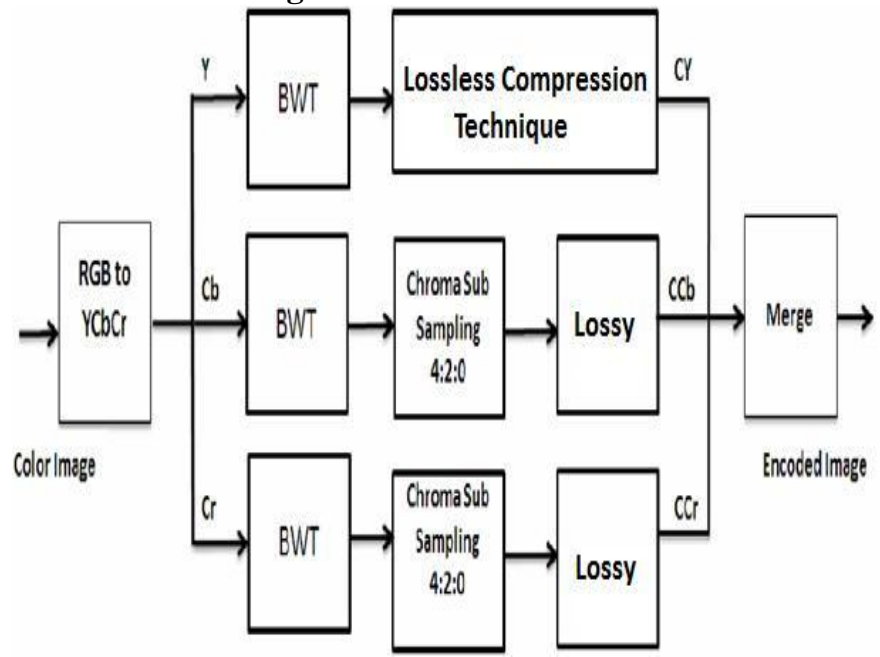

Figure-1: Block diagram for encoding of the proposed method

The given color image (RGB) is translated into $\mathrm{YCbCr}$ color space. All these components are decomposed using binary wavelet transform as stated in ${ }^{1}$. Chroma sub sampling of 4:2:0 is done for decomposed $\mathrm{Cb}, \mathrm{Cr}$ components which are followed by lossy mode compression. Decomposed $\mathrm{Y}$ components are subjected to lossless technique without sub-sampling. The resultant data tables are merged together to form the entire data table and the binary plane forming encoded image. The decoding procedure is shown in the figure 2

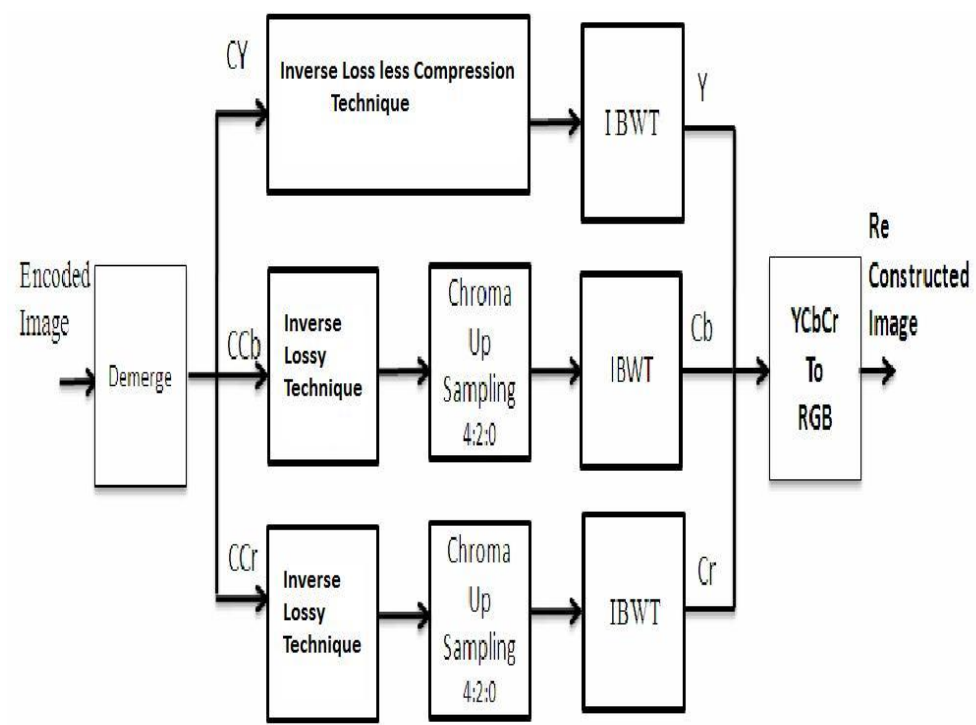

Figure 2: Block Diagram for decoding of the proposed method

\section{Procedure Main:-}

\section{BEGIN}

callConvertToYCbCr ( )

// dividing the image into $\mathrm{Y}, \mathrm{Cb}, \mathrm{Cr}$ formats

Call Raster_Line_Compress ()

// generate bit plane and data tables

Call Raster_Line_Merge ()

$/ /$ merge the bit plane and data table

Call lossless compression ()

END

\section{CONVERT_TO_YCbCr( )BEGIN}

//converting sourc image to $\mathrm{Y}, \mathrm{Cr}, \mathrm{CbO}$

pen source image File;
Open Y image file

Open $\mathrm{Cb}$ image file

Open $\mathrm{Cr}$ image file

$\mathrm{y} / /$ holds the y image pixel

$\mathrm{cb} / /$ holds the $\mathrm{cb}$ image pixel

$\mathrm{cr} / /$ holds the cr image pixel

a //holds image current pixel

$\mathrm{b} / /$ holds image next pixel

c // holds image next next pixel

While( (a=read(sours_image)!=eof)

BEGIN

$\mathrm{b}=$ read(sours_image) 
$\mathrm{c}=\mathrm{read}($ sours_image)

$\mathrm{y}=$ caluclateY using $\mathrm{a}, \mathrm{b}, \mathrm{c}$

$\mathrm{cb}=$ calculate $\mathrm{cb}$ using $\mathrm{a}, \mathrm{b}, \mathrm{c}$

$\mathrm{cr}=$ calculate cr using $\mathrm{a}, \mathrm{b}, \mathrm{c}$

write y to $\mathrm{Y}$ _image_file

write $\mathrm{cb}$ to $\mathrm{Cb}$ _image_file

write $c r$ to $\mathrm{Cr}$ _image_file

END

Close source image file

Close Y image file

Close $\mathrm{Cb}$ image file

Close $\mathrm{Cr}$ image file

END

PROCEDURE

Procedure Raster_Line_BPT_Compress ()

// subroutine generate bit plane and data table

/* Data Item Used */

Prev_pixel // holds previous pixel

Cur_pixel // holds current pixel

Bit_plane $/ * 8$ bit number to hold the status bit to indicate wether pixel is retained or not retained*/

\section{BEGIN}

Open raw image file

Open bit plane file

Open data table file

Cur_pixel=read(image)

Write cur_pixel to data table

Append bit 1 to bit_plane

Prev_pixel=cur_pixel

While((cur_pixel=read(image) $)$ !=eof $)$

\section{BEGIN}

$/ *$ if repeated consecutive pixel value append 0 to bit plane to indicate that pixel duplicate so not retained*/

if(cur_pixel=prev_pixel) then

append bit 1 to bit_plane

write cur_pixel to data table file

prev_pixel=cur_pixel

End

If bit_plane is full then

Write bit_plane to bitplane file

If bit_plane is not full then

Write bit_plane to bitplane file

Close raw image file

Close bitplane file

Close data table file
Procedure Raster_Line_BPT_Merge ()

$/ *$ to merge bit plane and data table files \& generate intermediate compressed file*/

/* Data item used*/

Cur_byte

Begin

Open bit plane file

Open data table file

Open bpds file

While((cur_byte=read(bitplane file) $) !=$ eof $)$

\section{BEGIN}

End

Write cur_byte to bpds file

While ((cur_byte=read(data table file $)) !=e o f)$

Begin

End

Write cur_byte to bpds file

\section{PROCEDURE LOSSLESS_COMPRESSION () BEGIN}

/*Design code for selecting image Compress compress buttons*/

Cur_Byte //current byte

Prv_Byte //Previous byte

No of bytes //caluclating the no. of bytes BEGIN

Open source image file

Open data_table file

Open bit_plane file

Cur_byte=read from source image file

Prv_byte=cur_byte

Write Cur_byte to data_table

while $(($ cur_byte $=$ read source image $)$ !eof $)$

\section{BEGIN}

bpData $=$ (byte) left shift the bit_plane data compare cur_bytewith prv_byte

then

write cur_byte to data_table

bit_data $=0$

prv_Byte=cur_Byte;

increment no of Bits with

compare if no of Bits $=8$

then

write bit_data to bit_plane file

no of Bits=0

bpData $=0$

increment bpCount

END

End

\section{PROCEDURE}

Results:-

For this, an experimental analysis is one with the different raw images whose resolution is 128x128. Gruop 1 level based binary wavelet transform decomposition is implemented. The results obtained with the different thresholding values are tabulated. 


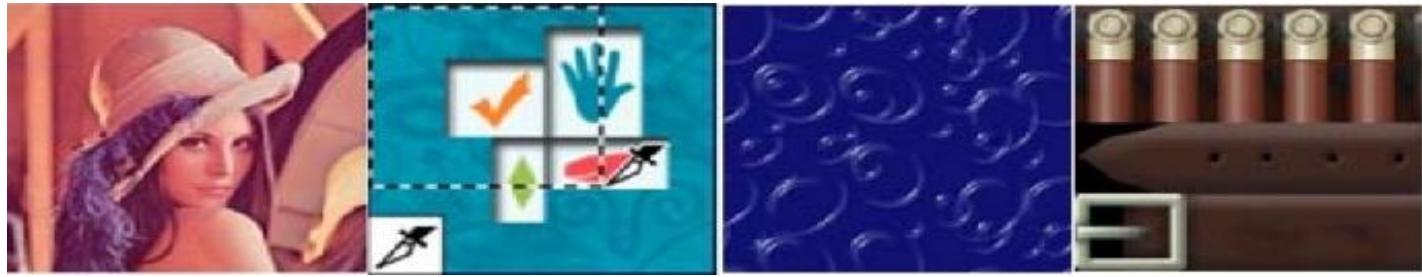

Figure 3: Sample test images for the experiment

STEP BY STEP ALGORITHM EXECUTION
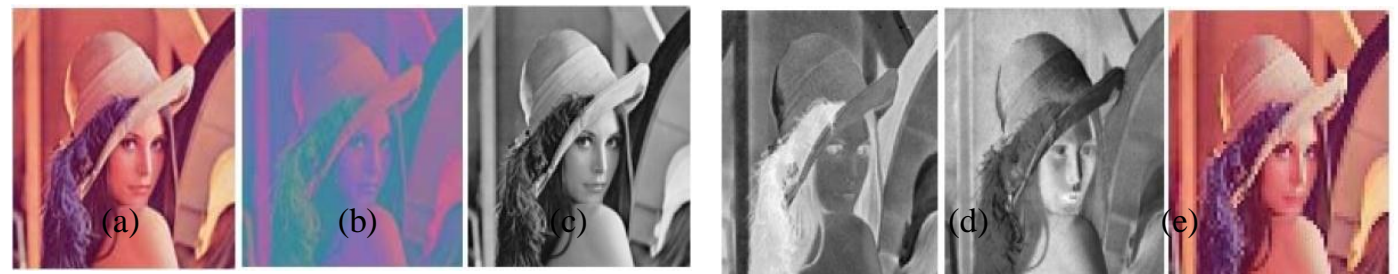

Figure 4: (a) Original image (b) YCbCr image (c) Y-component (d) Cb-component (e) Cr-component (f) Restored image with threshold 8 of $\mathrm{CR}=1.8895$ and $\mathrm{PSNR}=32$

In this method of encoding sub sampling of 4:2:0 is done for $\mathrm{Cb}$ and $\mathrm{Cr}$ components followed by lossy coding. Thus, obtained encoded stream is merged together to form a compressed set of the given image. The encoded image is again split up into $\mathrm{Y}, \mathrm{Cb}$ and $\mathrm{Cr}$ components which are by chroma up sampling. The inverse BWT is applied for details to restore them back to $\mathrm{Y} \mathrm{Cb}$ and $\mathrm{Cr}$ components. These are again reconverted back into RGB to get a best view

TABLE 1: ANALYSIS OF COMPRESSION RATIO FOR VARIOUS THRESHOLD VALUES

\begin{tabular}{|c|c|c|c|c|c|}
\hline \multicolumn{7}{|c|}{ THRESHOLD VERSUS CRR } \\
\hline & TH4 & TH8 & TH16 & TH32 & TH64 \\
\hline T32 & 2.5039 & 3.789 & 5.97 & 10.38 & 16.009 \\
\hline WIND & 1.6946 & 2.6768 & 4.3678 & 4.8998 & 51.125 \\
\hline SOAP & 6.0339 & 19.5771 & 19.897 & 34.075 & 34.148 \\
\hline LENNA & 1.4595 & 1.8895 & 2.6125 & 3.6987 & 5.985 \\
\hline SANTA & 2.711 & 3.6171 & 35.6562 & 58.524 & 58.5245 \\
\hline KODIM & 2.384 & 3.251 & 4.7123 & 6.9357 & 9.7632 \\
\hline COFFEBEEN & 1.7054 & 2.6043 & 4.7096 & 34.077 & 51.0995 \\
\hline HWA & 1.9378 & 2.5318 & 3.1032 & 3.5504 & 4.2304 \\
\hline
\end{tabular}

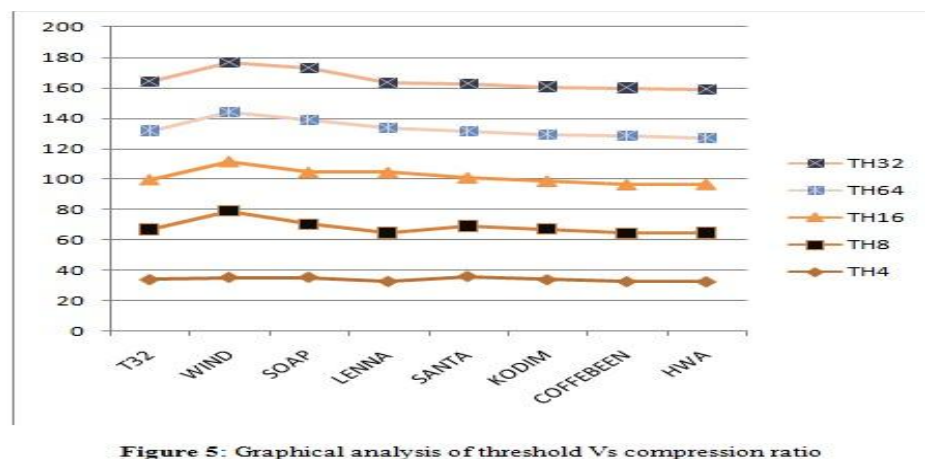

TABLE 2: ANALYSIS OF THRESHOLD VALUE AND PSNR FOR DIFFERENT IMAGES

\begin{tabular}{|c|c|c|c|c|c|}
\hline \multicolumn{7}{|c|}{ THRESHOLD VERSUS PSNR } \\
\hline & TH4 & TH8 & TH16 & TH32 & TH64 \\
\hline T32 & 33.716 & 33.156 & 32.74 & 32.26 & 32.2455 \\
\hline WIND & 35.2303 & 43.4356 & 32.9 & 32.5887 & 32.543 \\
\hline SOAP & 35.309 & 34.768 & 34.356 & 34.2565 & 34.4 \\
\hline
\end{tabular}




\begin{tabular}{|c|c|c|c|c|c|}
\hline LENNA & 32.5345 & 32.00678 & 39.78 & 29.456 & 29.452 \\
\hline SANTA & 35.8764 & 33.234 & 31.7654 & 31.3345 & 30.6579 \\
\hline KODIM & 33.6578 & 33.316 & 31.6789 & 31.37654 & 30.7689 \\
\hline COFFEBEEN & 32.3545 & 31.9987 & 31.8976 & 31.9234 & 31.92 \\
\hline HWA & 32.2908 & 32.1789 & 32.1032 & 32.0255 & 30.4624 \\
\hline
\end{tabular}

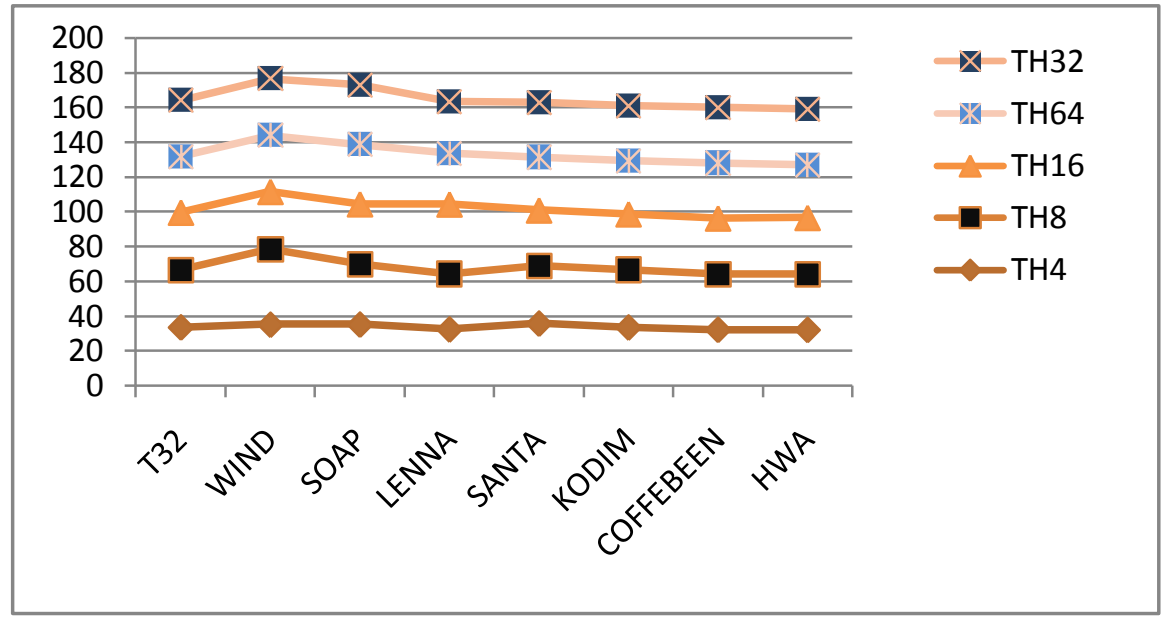

Figure 6: Graphical analysis of threshold Vs PSNR

\section{Conclusion and Recommendations:-}

Compressing the color images efficiently is one of the major problems in multimedia applications. So we have tested the efficiency of color image compression using BWT algorithm. The Lossless algorithm is applied for Y (luminance) and Lossy for $\mathrm{Cb}, \mathrm{Cr}$ (chrominance) of color image. This work may be extended for better compression by applying Huffman and arithmetic coding. The Lossy produces much higher compression rate than all the three techniques but introduces little loss. The loss is visually insignificant when the threshold value is 4 or 8 . When the threshold is 16 or 32 the loss is visually observable. The memory requirements for processing the images in all of these techniques are significantly less compared to JPEG. The JPEG technique requires more memory because the entire image needs to be brought into memory. Proposed method require no complex calculations and processing of the data is performed only in terms of integers, so there is no chance of loss of precision. The JPEG technique requires complex calculations. The processing is done in terms of real numbers where there is possibility of loss of precision.

\section{References:-}

[1]. M.Ashok, Dr.T.Bhaskar Reddy, Dr.B.Eswara Reddy,"Binary wavelet transform for image representation”,IJEST,vol3,no.8,Aug 2011,pg.no:6286-6290.

[2]. Rafael C.Gonzalez, Richard E.woods "Digital image processing " second edition Pearson education, printiceHall

[3]. W.S.lee "Edge adaptive predication for loss less image coding "in proc. IEE data CompressionConference, Snowbird Utah march 199,pp 483-490

[4]. Skodras ,A.C. Christopoulis and T.Ebrahim ,2001 .The JPEG 2000 still image compression standardIEEE signal Proc. Mag 18(5) : $36-58$

[5]. Sadashivappa et al "Color image compression using SPIHT Algorithm" international journal of computer Applications ( 0975-8887) Vol 16-No 7 February 2011.

[6]. Noise to mark ratio minimization by weighted non-nagative matrix factorization, Joonas NiKunen et al ICASSP 2010 organised by IEEE.

[7]. Image Denoising based on wavelet and Multifractals for singularitydetection Junmie Zhong andRoula Ning IEEE transaction on image processing Vol 14 No:10 oct 2005.

[8]. I. Pitas Digital Image Processing algorithms and Applications Wiely 2000.

[9]. Digital Image Processing using MAT Lab R.C. Gonzalez and et al 2004 Pearson Education. 